\title{
El efecto mediador de la RSE en la relación de la orientación emprendedora y el desempeño de las cooperativas agroalimentarias
}

\author{
Felipe Hernández Perlines
}

\section{RESUMEN}

Este trabajo analiza el efecto mediador de la responsabilidad social de la empresa en la relación entre la orientación emprendedora y el desempeño de las cooperativas agroalimentarias. Este objetivo ya supone una gran aportación en sí mismo. La segunda de las aportaciones de este trabajo es la utilización de una triple vía para la obtención de los datos: un cuestionario que se envió vía mail sobre orientación emprendedora a cooperativas agroalimentarias de España entre enero y abril de 2015 (112 cuestionarios válidos); el análisis de las memorias de sostenibilidad (68 memorias) y los datos económicos a partir de la base de datos SABI. Para la contrastación de los datos se utilizó el programa de ecuaciones estructurales Smartpls 3.2.3. La conclusión más destacada de esta investigación es que se constata el efecto mediador de la RSE en la relación entre la orientación emprendedora y el desempeño.

PALABRAS CLAVE: Responsabilidad social de la empresa, orientación emprendedora, cooperativas agroalimentarias, PLS.

CLAVES ECONLIT: M140, L310, Q130, L120.

Cómo citar este artículo: HERNÁNDEZ-PERLINES, F. (2015): "El efecto mediador de la RSE en la relación de la orientación emprendedora y el desempeño de las cooperativas agroalimentarias", CIRIEC-España, Revista de Economía Pública, Social y Cooperativa, 85, 217-244.

Correspondencia: Felipe Hernández-Perlines, Universidad de Castilla-La Mancha. E-mail: Felipe.HPerlines@uclm.es. 


\title{
L'effet médiateur de la RSE dans la relation entre l'orientation entrepreneuriale et la performance des coopératives agroalimentaires
}

RÉSUMÉ : Cet article analyse l'effet médiateur de la responsabilité sociale des entreprises dans la relation entre l'orientation entrepreneuriale et la performance des coopératives agroalimentaires. Cet objectif constitue déjà une contribution majeure en soi. La seconde contribution de ce travail est l'utilisation d'une voie triple pour la collecte de données : un questionnaire envoyé par e-mail sur l'orientation entrepreneuriale des coopératives agroalimentaires en Espagne entre janvier et avril 2015 (112 questionnaires validés), l'analyse des rapports sur le développement durable (68 rapports) et les données économiques de la base de données SABI. Le programme d'équations structurelles Smartpls 3.2.3 a servi au contraste des données. La conclusion principale de cette recherche est que l'on peut observer l'effet médiateur de la RSE dans la relation entre l'orientation entrepreneuriale et sa performance.

MOTS CLÉ : Responsabilité sociale des entreprises, orientation entrepreneuriale, coopératives agroalimentaires, PLS.

\section{The mediating effect of CSR on the relationship between entrepreneurial orientation and performance in agricultural cooperatives}

\begin{abstract}
This paper analyses the mediating effect of a company's social responsibility on the relationship between entrepreneurial orientation and performance in agricultural cooperatives. This objective constitutes a major contribution in itself. The second contribution made by this paper is the use of three sources of data: a questionnaire on entrepreneurial orientation mailed to agricultural cooperatives in Spain between January and April 2015 (112 valid questionnaires), an analysis of sustainability reports (68 reports), and financial data from the SABI database. The SmartPLS 3.2.3 structural equations program was used to test the data. The key finding of this research is that CSR has a mediating effect on the relationship between entrepreneurial orientation and performance.
\end{abstract}

KEY WORDS: Corporate social responsibility, entrepreneurial orientation, agri-food cooperatives, PLS. 


\section{EL EFECTO MEDIADOR DE LA RSE EN LA RELACIÓN DE LA ORIENTACIÓN EMPRENDEDORA Y EL DESEMPEÑO DE LAS COOPERATIVAS AGROALIMENTARIAS}

\section{1.- Introducción}

Este trabajo se justifica por el hecho que la RSE está presente en la esencia misma del cooperativismo, convirtiéndose en un factor clave para las mismas. En los últimos años, asistimos a un creciente interés por la RSE (Gallardo-Vázquez et al., 2013). En este sentido, la RSE se ha convertido en uno de los objetivos con más peso en la toma de decisiones (Nieto y Fernández, 2004; De La Cuesta, 2004; Garriga y Melé, 2004). Aunque podemos encontrar trabajos en la literatura que afirman que la RSE afecta a los resultados de las empresas (Orlitzky et al., 2003; Allouche y Laroche, 2005; Wu, 2006), todavía no está muy clara la relación causa-efecto entre ambos aspectos. Por eso, resulta de interés tratar de analizar el papel estratégico que posee la RSE en las empresas, en general, y en las cooperativas agroalimentarias, en particular. Son numerosos los trabajos que sostienen que un comportamiento responsable de las empresas contribuye a crear valor a partir de los recursos estratégicos que aquella posea (Surroca et al., 2010; Wang y Bansal, 2012).

Por otro lado, la orientación emprendedora es una de las áreas de investigación donde más se está desarrollando un cuerpo acumulativo de conocimientos, recibiendo una considerable atención conceptual y empírica (Basso et al., 2009; Rauch et al., 2009; Rodrigo-Alarcón et al. 2013; Covin y Miller, 2014; Hernández Perlines, 2014). Si efectuamos una revisión de la literatura sobre orientación emprendedora, observamos que la mayoría de los trabajos se centran en analizar el efecto de la orientación emprendedora en el desempeño empresarial. A la hora de definir la orientación emprendedora, la perspectiva de Shumpeter (1934) resulta ser un punto de partida y de referencia en las propuestas de su conceptualización. Podemos afirmar que los orígenes orientación emprendedora están ligados al concepto de emprendimiento corporativo. El emprendimiento corporativo puede albergar dos formas diferentes: como una iniciativa empresarial o como un emprendimiento estratégico (Morris et al., 2012). El emprendimiento estratégico puede ser entendido como la búsqueda de oportunidades (Ireland et al., 2003) con el fin último de crear una ventaja competitiva (Kuratko y Audretsch, 2009). Según Aloulou y Fayolle (2005) se trata de la disponibilidad de la empresa para emplear comportamientos emprendedores. Cuando hablamos de orientación emprendedora estamos haciendo referencia a un conjunto de procesos, prácticas y actividades relacionadas con la toma de decisiones que posibilitan la actividad emprendedora (Dess y Lumpkin, 2005; Covin y Slevin, 1991; Lumpkin y Dess, 1996; Miller, 1983), siendo un instrumento fundamental para el logro del éxito empresarial en términos de crecimiento, rentabilidad y creación de empleo (Bosma y Levie, 2010). En definitiva, la orientación emprendedora permite establecer un marco adecuado para analizar la actividad emprendedora de la empresa (Naldi et al., 2007). 
En este trabajo pretendemos analizar cómo la RSE afecta a la relación entre la orientación emprendedora y el desempeño de las cooperativas agroalimentarias. Este análisis lo haremos considerando tanto el efecto conjunto de cada una de las dimensiones que definen la orientación emprendedora (innovación, proactividad y asunción de riesgos) como de cada una de ellas por separado.

La oportunidad de este trabajo está en que no existen estudios que traten de analizar el efecto mediador de la RSE en la relación entre la orientación emprendedora y el desempeño empresarial en las empresas en general, y mucho menos, en las cooperativas agroalimentarias.

Los datos se han obtenido de un cuestionario enviado a los presidentes de Consejos Rectores de cooperativas agroalimentarias ubicadas en diferentes partes del territorio nacional. El trabajo de campo se realizó entre los meses de enero a abril de 2015. Para la contrastación de las hipótesis planteadas se ha utilizado el programa estadístico smartpls 3.2.1.

El presente trabajo se estructura de manera que tras esta introducción se realiza una revisión sobre orientación emprendedora y sobre RSE en la literatura. Posteriormente, nos adentramos en la parte metodológica, en la que tras el planteamiento de la investigación en el que se diseña el modelo y se enuncian las hipótesis. Después, explicamos cómo se ha efectuado la recogida de información y cómo se han medido las variables. En tercer lugar, analizamos los resultados obtenidos y dedicamos un espacio a la discusión de los mismos. Finalmente, exponemos las conclusiones más relevantes a las que hemos llegado.

\section{2.- Marco teórico}

\subsection{Orientación emprendedora}

En los últimos años encontramos una gran cantidad de estudios que se centran en analizar la orientación emprendedora. Dicho concepto nos permite determinar el grado de emprendimiento, y además, se centra en el emprendimiento a nivel de empresa. La mayoría de los trabajos, se centran en analizar el efecto de la orientación emprendedora en el desempeño empresarial. A la hora de definir la orientación emprendedora, la perspectiva de Shumpeter (1934) resulta ser el punto de partida y de referencia en las propuestas de su conceptualización. Cuando hablamos de orientación emprendedora estamos haciendo referencia a un conjunto de procesos, prácticas y actividades relacionadas con la toma de decisiones que posibilitan la actividad emprendedora (Dess y Lumpkin, 2005; Covin y Slevin, 1991; Lumpkin y Dess, 1996; Miller, 1983), siendo un instrumento fundamental para el logro del éxito empresarial en términos de crecimiento, rentabilidad y creación de empleo (Bosma y Levie, 


\section{EL EFECTO MEDIADOR DE LA RSE EN LA RELACIÓN DE LA ORIENTACIÓN EMPRENDEDORA Y EL DESEMPEÑO DE LAS COOPERATIVAS AGROALIMENTARIAS}

2010). En definitiva, la orientación emprendedora permite establecer un marco adecuado para analizar la actividad emprendedora de la empresa (Naldi et al., 2007).

Miller (1983) fue el primero en hablar de la orientación emprendedora, afirmando que "una firma emprendedora es aquella que se dedica a la innovación del producto o mercado, acomete actividades un tanto arriesgadas y es la primera en alcanzar innovaciones "proactivas», adelantando a sus competidores" (Miller, 1983, p. 771). Más tarde, Covin y Slevin (1989) completaron la anterior definición afirmando que la orientación emprendedora de una empresa depende del grado de ésta para favorecer el cambio y la innovación, para asumir riesgos y competir agresivamente (Miller y Friesen, 1983; Covin y Slevin, 1991; Knight, 1997; Wiklund y Shepherd, 2005).

En este trabajo nos hemos decantado por la definición de orientación emprendedora que incluye la innovación, proactividad y la asunción de riesgos de Miller (1983). La innovación se refiere a la capacidad de la empresa para apoyar nuevas ideas y la experimentación, para introducir nuevos productos y el uso de procesos creativos (Miller y Friesen, 1983; Kropp et al., 2006; Chandra et al., 2007). Por otro lado, la proactividad, se refiere a la búsqueda de la ventaja del pionero, mediante la anticipación a los deseos y necesidades futuras del mercado (Covin y Slevin, 1989; Lumpkin y Dess, 1996). Finalmente, asunción de riesgos conlleva la puesta en marcha de acciones audaces que comprometen significativos niveles de recursos (Lumpkin y Dess, 1996).

La mayoría de los trabajos de investigación se centran en analizar el efecto de la orientación emprendedora en el desempeño empresarial. Además, se considera que la orientación emprendedora es un proceso (Hult et al., 2003) organizativo fundamental que contribuye a reafirmar el rendimiento de la empresa (Miller, 1983). Sobre la base de esta discusión, ponemos a prueba que la orientación emprendedora influye positivamente en el desempeño de las cooperativas agroalimentarias y enunciamos la siguiente hipótesis:

$H_{1}$ : La orientación emprendedora afecta positivamente al desempeño empresarial de las cooperativas agroalimentarias.

Diversos trabajos previos estudian el efecto mediador de algunas variables en la relación entre la orientación emprendedora y el desempeño empresarial. Autores como Miller (1983), Lumpkin y Dess (1996) y Runyan et al. (2008), entre otros, analizan el efecto mediador de la estructura organizativa en la influencia de la orientación emprendedora en el desempeño empresarial. También encontramos el efecto mediador de la estrategia en dicha relación en trabajos de autores como Álvarez y Busenitz (2001), Moreno y Casillas (2008) y Galán y Sánchez-Bueno (2009). Otro grupo de trabajos como los de Dimitratos et al. (2004), Zahra et al. (2000) y Yli-Renko et al. (2001) consideran el efecto mediador del tamaño en la relación entre la orientación emprendedora y el desempeño empresarial. Finalmente, encontramos trabajos que consideran el efecto de la antigüedad de la empresa en la relación entre la orientación emprendedora y el desempeño empresarial. Entre estos destacan los de Zahra et al. (2000), Etchebarne et al. (2010) y Kuivalainen et al. (2010). Si bien no encontramos en la litera- 
tura trabajos específicos que relacionen la orientación emprendedora con actuaciones de RSC o sostenibilidad, algunos estudios sugieren un impacto positivo de la orientación emprendedora en la RSE (Mickiewicz et al., 2010, Schaper y Savery, 2004). Este trabajo pretende resolver este gap planteando la siguiente hipótesis:

$\mathrm{H}_{2}$ : La orientación emprendedora influye positivamente en la RSE de las cooperativas agroalimentarias.

\subsection{Responsabilidad social}

En este apartado vamos a realizar una revisión de los aspectos teóricos más destacados de la RSE y de la orientación emprendedora. Empecemos por el primero. No cabe duda que en los últimos años ha crecido la importancia de la RSE como objetivo a tener en cuenta por las empresas (Nieto y Fernández, 2004; De La Cuesta, 2004; Garriga y Melé, 2004). Aspectos como la globalización, la internacionalización de los mercados y su transparencia, el impacto medioambiental de determinadas actividades ponen de manifiesto la creciente preocupación de las empresas por la RSE (De la Cuesta y Valor, 2003).

Este mayor interés por la RSE mostrado por las empresas se deriva de su consideración como herramienta estratégica de gestión (Carroll, 1999; Porter y Kramer, 2006), siendo considerada como una inversión que permite a las empresas lograr una ventaja competitiva sostenible a partir de capacidades únicas y distintivas (Burke y Logsdon, 1996; Husted y Allen, 2000; Mc Williams y Siegel, 2001; Toro, 2006).

La sociedad actual demanda que las empresas busquen valor para todos los grupos de interés que interactúan con ella, por lo que la RSE pasa a ser parte de la gestión de las empresas con la finalidad de generar confianza y mejorar la reputación de la empresa (De La Fuente y De Quevedo, 2003).

Los diferentes estudios que podemos encontrar en la literatura han ido evolucionando desde trabajos que tratan de dar una definición de la RSE y de cómo y por qué las empresas la implantan, hasta trabajos que intentan explicar el efecto de la RSE en los resultados a largo plazo de las empresas (Waddock y Graves, 1997; Tsotsoura, 2004; De La Cuesta, 2004; Porter y Kramer, 2006; Hull y Rothenberg, 2008).

Existen múltiples definiciones de RSE. Una que parece estar muy extendida es la de Carroll (1979). Para este autor la RSE "abarca todas las expectativas económicas, legales, éticas y discrecionales que la sociedad tiene de las organizaciones en un momento dado". Por su parte, el Consejo Mundial de los Negocios para el Desarrollo Sostenible (1999) define la RSC de una manera más concreta, haciendo hincapié en el desarrollo sostenible y en la participación de los diferentes grupos de interés. 


\section{EL EFECTO MEDIADOR DE LA RSE EN LA RELACIÓN DE LA ORIENTACIÓN EMPRENDEDORA Y EL DESEMPEÑO DE LAS COOPERATIVAS AGROALIMENTARIAS}

En el Documento Final de la Cumbre Mundial de las Naciones Unidas (2005) se pone de manifiesto tres cuestiones fundamentales acerca del concepto de RSC, como son: los stakeholders, la voluntariedad y el concepto de desarrollo sostenible. Posteriormente Dahlsrud (2008) definió la RSE a partir de cinco aspectos: los grupos de interés o stakeholders, la voluntariedad, la dimensión social, la dimensión económica y la dimensión medioambiental. Estas tres últimas dimensiones son las que según Husillos et al. (2009) se contienen en el concepto de desarrollo sostenible del Documento de Naciones Unidas antes mencionado.

Por otra parte, también existe en la literatura académica un interesante debate acerca de si la RSE debe ser incorporada a la legislación o en cambio, deben ser unas acciones de carácter voluntario llevadas a cabo por las organizaciones (Carroll y Shabana, 2010). Así, por un lado, un grupo de autores, encabezados por Williamson et al. (2006), piden una mayor regulación de las acciones de RSE, debido a que no confían en los mecanismos de mercado para su regulación. Por otro, encontramos a aquellos autores que se oponen a la regulación de las acciones de RSE, que siguen abogando porque la RSE son acciones voluntarias y que deben ir más allá de las responsabilidades legales de las empresas (Carroll 1991; Phillips et al., 2003).

Si nos centramos en la RSE de las cooperativas, ésta ha ido adquiriendo una nueva dimensión, aunque no es nada nuevo para ellas. La RSE es una forma de gestión con mayor compromiso frente a la sociedad y esta muy presente en las cooperativas, como lo puso de manifiesto la Comisión Europea (2002), cuando indicó que "las cooperativas tienen una larga tradición en combinar viabilidad económica y responsabilidad social, gracias al diálogo entre las partes interesadas y a la gestión participativa, y pueden servir de referencia a otras organizaciones".

No cabe duda que las cooperativas agroalimentarias son una forma de empresa que tienen su fundamento en los valores de la RSE, puesto que poseen un comportamiento ético y un conjunto de valores sociales (Castro, 2006). Además, siguiendo a Server y Capó (2009), porque este tipo de entidades desarrollan acciones que pueden enmarcarse en la solidaridad e inserción social, la cohesión territorial, etc.

Las cooperativas agroalimentarias, como entidades de economía social, además de perseguir sus fines económicos poseen unas responsabilidades de tipo social y medioambiental. Por lo tanto, cumplen perfectamente con las tres dimensiones de la RSE (Puentes y Velasco, 2009).

Si analizamos los valores y principios cooperativos enunciados por la $\mathrm{ACl}$ (Alianza Cooperativa Internacional) en 1995, observamos que muchos coinciden con la RSE. En este sentido, podemos afirmar que tanto la RSE como los valores cooperativos poseen puntos iniciales comunes (Carrasco, 2007). Además, los valores cooperativos son valores que entroncan directamente con la RSE (Melian, 2006), pudiendo considerar a las cooperativas como una unidad exponente de los principios de RSE (Mozas y Puentes, 2010). 
Si nos remontamos al inicio del cooperativismo, el movimiento cooperativo ha sido pionero en el desarrollo y la puesta en práctica de la RSE. No tenemos más que ver las características propias de las cooperativas: estructura democrática, valores de autoayuda, equidad, solidaridad, relevancia de sus miembros, servicio al interés de sus trabajadores, de la comunidad y del entorno en el que operan. Por lo tanto, la RSE no es un descubrimiento nuevo para las cooperativas: los valores y principios de la RSE han estado presentes en el corazón del modelo cooperativo desde sus orígenes.

El desarrollo de la RSE puede ser más fácil en las cooperativas porque en ellas se cumple la doble o triple condición de socio (propietario, proveedor y/o cliente, directivo), lo que permite que el equilibrio entre los diferentes grupos de interés ( $T^{\mathrm{a}}$ de los stakeholders, Vargas y Vaca, 2005).

Se observa como cada vez más las cooperativas están dando a conocer como en su actividad cotidiana está presente la RSE, hasta tal punto que podemos afirmar que tienen mucho camino andado por su propia naturaleza y por su funcionamiento socialmente responsable, lo que las sitúa en una inmejorable posición estratégica en este ámbito (Collado, 2006; Server et al., 2009).

Son múltiples los trabajos que podemos encontrar en la literatura que tratan de analizar la relación entre la RSE y el RE. La mayoría de ellos poseen como punto común que utilizan modelos lineales para analizar la relación entre la RSE y el desempeño empresarial. Si embargo, si efectuamos una revisión más profunda de la literatura, podemos encontrar trabajos que por no encontrar vínculos simples en la relación entre la RSE y el RE emplean modelos no lineales: son los denominados modeIos en "U" invertida (Bowman y Haire, 1975; Sturdivant y Ginter, 1997; Lankoski, 2000; Moore, 2001; Maron, 2006; Barnett y Salomon, 2006, 2012).

Coincidimos con Alvarado et al. (2011) al afirmar que la mayoría de los trabajos que tratan de analizar la influencia de la RSE en el desempeño empresarial concluyen que la RSE es una fuente de ventaja competitiva y de creación de valor para la empresa. Además, Gómez (2008) después de una profunda revisión de 140 estudios empíricos llega a la conclusión de la existencia de una relación positiva entre la RSE y el desempeño empresarial. En este trabajo seguimos el enfoque del impacto social, que establece que la adopción de medidas de RSE influye positivamente en el desempeño empresarial (García-Castro et al., 2010; Miras et al., 2011). Así, niveles más altos (bajos) de RSE conducen a niveles más altos (bajos) de desempeño empresarial (Waddock y Graves, 1997; Orlitzky et al., 2003; De la Cuesta, 2004; Nieto y Fernández, 2004; Allouche y Laroche, 2005; Porter y Kramer, 2006). Bajo este prisma podemos enunciar la tercera de las hipótesis del modelo que queremos contrastar:

$H_{3}$ : La adopción de medidas de RSE en todas sus dimensiones tiene una influencia positiva en el desempeño empresarial de las cooperativas agroalimentarias. 


\section{3.- Metodología}

\subsection{Planteamiento de la investigación}

Como comentamos en la introducción, el objetivo de este trabajo es doble: por un lado, analizar la influencia de la orientación emprendedora en el desempeño empresarial de las cooperativas agroalimentarias. Y por otro, comprobar el efecto mediador de la RSE en la relación entre la orientación emprendedora y el desempeño empresarial.

El modelo que proponemos es el resultado de una profunda revisión de la literatura y de la creencia de que la orientación emprendedora influye en las tres dimensiones de la RSE, y ésta a su vez, afecta al desempeño empresarial de las cooperativas agroalimentarias. En definitiva, la orientación emprendedora actúa como un antecedente de la RSE. Por otro lado, la RSE actúa como mediadora en la relación entre la relación emprendedora y el RE. El modelo propuesto puede verse en la figura 1.

\section{Figura 1. Modelo de efecto mediador de la RSE en la influencia de la orientación emprendedora en el desempeño empresarial}

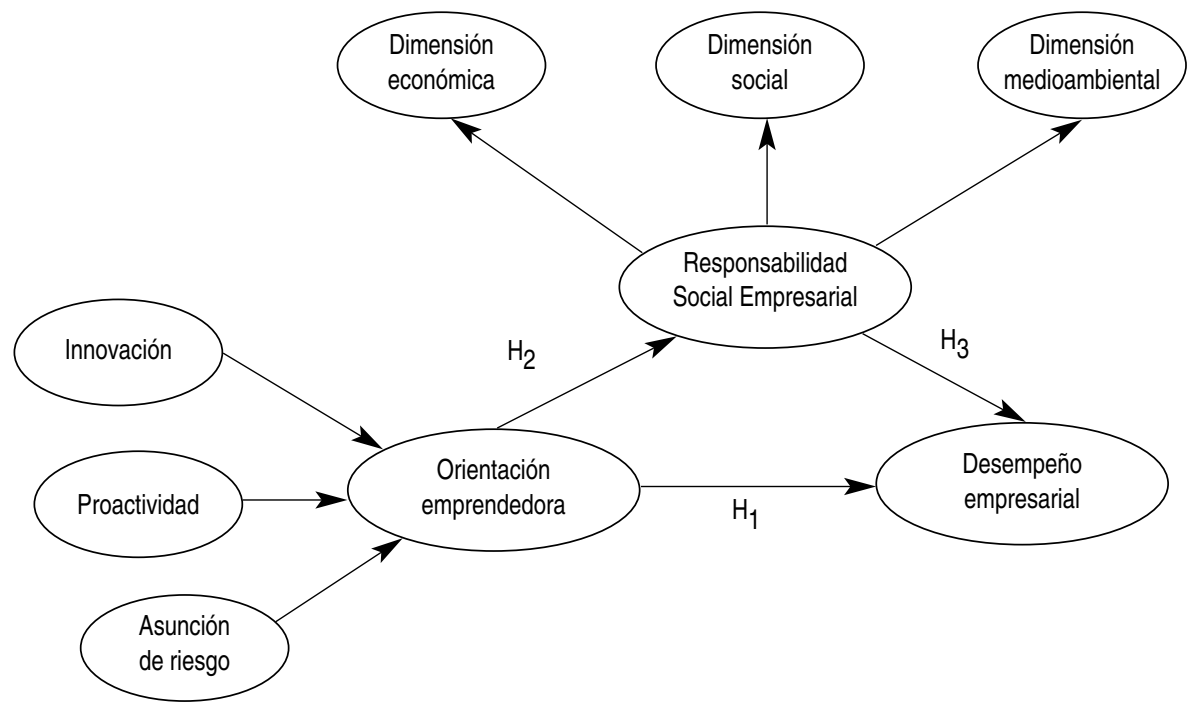

FUENTE: Elaboración propia. 


\subsection{Cuestionario y trabajo de campo}

En este trabajo nos hemos centrado en las cooperativas agroalimentarias, siendo este aspecto un punto fuerte de la investigación, ya que al ser un único tipo de cooperativas que los datos sean homogéneos (Mishra y Suar, 2010; Martínez-Campillo et al., 2013) y hace posible controlar ciertos factores de contingencia (Lyon et al., 2000; Rauch et al., 2009).

Los datos han sido obtenidos a través de tres vías. Los datos relativos a la orientación emprendedora han sido obtenidos a partir de una encuesta enviada a cooperativas agroalimentarias de todo el territorio nacional. Tras la revisión de la literatura sobre orientación emprendedora y desempeño empresarial se elaboró un borrador preliminar del cuestionario. En segundo lugar, para asegurar la validez de contenido del mismo, se desarrolló un proceso de discusión y reflexión con algunos expertos en las materias tratadas en el mismo, según la propuesta de Conca et al. (2004). A continuación, para garantizar que los items considerados en el cuestionario fueran plenamente comprensibles, se llevó a cabo un pre-test a presidentes de Consejos Rectores de cooperativas agroalimentarias. Las encuestas se hicieron mediante entrevista personal a cada uno de los encuestados ( 9 en total en esta fase de pre-test). Una vez superada esta fase, se procedió al envío del cuestionario por mail. La encuesta se realizó entre enero y abril de 2015. Al ser contestado el cuestionario por el presidente del Consejo Rector se aplicaron las directrices y recomendaciones de Huber y Power (1985) para evitar el sesgo en las respuestas de un único informante. En los diferentes items de percepción se ha utilizado una Likert 1-7 ( $1=$ totalmente en desacuerdo; $7=$ totalmente de acuerdo).

Dado que no obtuvimos información de todas las empresas que conformaban la población objeto de estudio, comprobamos la representatividad de la muestra y el sesgo de no respuesta a partir de variables con valores conocidos para la población total, como el sector y el número de empleados (Armstrong y Overton, 1977). Los análisis indicaron que no había diferencias significativas entre las empresas que contestaron y las que no lo hicieron, puesto que se considera que las empresas que responden más tarde son más parecidas a las que no responden (Armstrong y Overton, 1977), se realizó una comparación entre los primeros cuestionarios recibidos y los últimos. En ninguna de las variables se observó que hubiera diferencias significativas entre estos dos grupos de empresas. Después de los tres meses que duró el estudio de campo, obtuvimos 112 cuestionarios válidos y completos.

La segunda vía de obtención de datos ha sido a través de las memorias de sostenibilidad de las cooperativas agroalimentarias. Estas memorias de sostenibilidad han sido elaboradas conforme a los criterios del GRI. De las 112 empresas que habían contestado correctamente el cuestionario sobre orientación emprendedora, 68 también poseían memorias de sostenibilidad.

La tercera vía para la obtención de datos, sobre todo los de carácter financiero, ha sido la base de datos SABI (Sistema de Análisis de Balances Ibéricos). 


\section{Tabla 1. Ficha técnica del trabajo de campo del cuestionario}

$\begin{array}{ll}\text { Tamaño de la población } & 3.397^{1} \\ \text { Ámbito de aplicación } & \text { España } \\ \text { Respuestas obtenidas } & 112 \\ \text { Procedimiento muestral } & \text { Aleatorio simple } \\ \text { Nivel de confianza } & 95 \%, p=p=50 \% ; \alpha=0.05 \\ \text { Trabajo de campo } & \text { Enero-Abril } 2015\end{array}$

\subsection{Medida de las variables}

Orientación emprendedora: para medir la orientación emprendedora hemos utilizado tres dimensiones: innovación, proactividad y asunción de riesgos, según la escala propuesta en estudios previos de Miller (1983) modificada por Covin y Slevin (1989) y utilizada por autores como Knight y Cavusgil (2005); Sundqvist et al. (2012); Hernández Perlines (2014).

Responsabilidad social de la empresa: para medir la responsabilidad social de la empresa hemos utilizado diferentes indicadores que parten de los índices del GRI (Global Reporting Initiative). Estos indicadores poseen dos ventajas: por un lado, aúnan los efectos de diferentes dimensiones y, por otro, permiten hacer comparaciones entre diferentes empresas. Además, estos índices reflejan el desempeño responsable de la empresa en relación con sus resultados económico-financieros (Griffin y Mahon, 1997; Margolis y Walsh et al., 2007). En concreto en este trabajo hemos considerado los indicadores agrupados es las siguientes dimensiones:

1 - Dimensión Económica - Los indicadores que se contienen en esta dimensión nos ofrecen información del impacto económico de la empresa en el entorno en el que opera (De la Cuesta, 2004). En concreto, en este trabajo se han utilizado los indicadores propuestos por Hernández-Perlines y Sánchez-Infante (2015): gastos de personal, compras, donaciones monetarias a la comunidad, impuestos pagados, reservas y dotaciones.

2 - Dimensión Social - Los indicadores que reflejan esta dimensión tratan de manifestar la preocupación social de la empresa por sus grupos de interés. Para esta dimensión se han utilizado los indicadores propuestos por Hernández-Perlines y Sánchez-Infante (2015), quienes agrupan los ítems de esta dimensión en cuatro grupos: generales (principales mercados, regional, nacional, internacional), social (número de empleados en la organización que son, mujeres, extranjeros, con minusvalías, con riesgo de exclusión), formación y sociedad (gasto en formación, donaciones no monetarias 
a la sociedad) y desempeño (certificaciones y premios obtenidos, grupos de interés que identifican y a los que se dirigen, canales de diálogo de la empresa, e iniciativas externas en las que colabora).

3 - Dimensión Medioambiental - Esta dimensión ha sido considerada porque la gestión medioambiental es una fuente relevante de ventaja competitiva (Chavan, 2005; Tomomi, 2010). Para esta dimensión se han utilizado los indicadores propuestos por Hernández-Perlines y Sánchez-Infante (2015) correspondientes a consumos energéticos, utilización de materiales de origen reciclado, realización de reciclado de productos por la empresa, práctica de iniciativas medioambientales.

Desempeño empresarial: En este trabajo hemos optado por medir el desempeño empresarial a partir de indicadores objetivos basados en las escalas utilizadas por Wiklund y Shepherd (2003); Chirico et al. (211) y Kraus et al. (2012).

\section{4.- Discusión y resultados}

Para realizar el análisis de los resultados y la contrastación de las hipótesis planteadas, hemos elegido la técnica del PLS (Partial Least Squares), a través del programa smartpls 3.21 basada en componentes y la varianza. El método del PLS es particularmente interesante en las primeras etapas del desarrollo de la teoría (Ringle, Wende, y Will, 2010; Gefen, Rigdon y Straub, 2011) o cuando los investigadores incluyen escalas que han sido probadas y validadas en trabajos anteriores o por el tamaño relativamente pequeño de la muestra (Barroso et al., 2005; Reinartz, Haenlein y Henseler, 2009) o finalmente, cuando los modelos son muy complejos (Hair, Hutl, Ringle y Sarstedt, 2014).

Antes de continuar con la aplicación del análisis del PLS, comprobamos el tamaño de la muestra. Para ello tomamos como válida la regla de Barclay, et al. (1995), basada en que la muestra requerida será la que sirva de base a la regresión múltiple más compleja que se pueda encontrar. Si se utiliza una regresión heurística de 10 casos por predictor, el cálculo se realizará como sigue: a) el número de indicadores de la escala con el mayor número de indicadores formativos, multiplicado por 10. b) el mayor número de variables latentes antecedentes que conducen a un constructo dependiente, multiplicado por 10. El constructo dependiente, que recibe más relaciones causales en el modelo es el constructo RSE, que son tres, por lo que nos bastaría una muestra de 30 casos para poder realizar el análisis. Los casos con los que se trabaja, como ya se ha indicado anteriormente son de 112, (68 si consideramos las cooperativas de las que tenemos memorias de sostenibilidad). Por lo tanto, el número de casos son suficientes para realizar el estudio y llegar a conclusiones generalizables. 


\section{EL EFECTO MEDIADOR DE LA RSE EN LA RELACIÓN DE LA ORIENTACIÓN EMPRENDEDORA Y EL DESEMPEÑO DE LAS COOPERATIVAS AGROALIMENTARIAS}

Una vez comprobado que la muestra es adecuada para la aplicación del PLS, pasamos a la interpretación y análisis del modelo. Para ello, se siguen dos etapas para asegurar que las escalas de medida propuestas son válidas y fiables (Barclay et al., 1995): 1) análisis del modelo de medida; 2) análisis del modelo estructural.

\section{Análisis y evaluación del modelo de medida para constructos de primer orden}

Con este análisis pretendemos comprobar si los conceptos teóricos son medidos correctamente a través de las medidas observadas. En el modelo que se ha plantados encontramos los siguientes constructos de primer orden:

1 - En la orientación emprendedora:

Innovación:

Proactividad

Asunción de Riesgos

2 - En la RSE:

La dimensión económica

La dimensión social

La dimensión medioambiental

3 - El desempeño empresarial

Lo primero que comprobamos la fiabilidad individual de los indicadores de los constructos de primer orden. Para que un indicador sea considerado como integrante de un constructo, aquel debe poseer una carga $(\lambda)>0,7$. Esto implica que la varianza compartida entre el constructo y sus indicadores es mayor que la varianza del error (Carmines y Zeller, 1979).

La dimensión económica de la RSE se han considerado un total de 6 indicadores, en la dimensión social se utilizaron 47 indicadores y en la dimensión medioambiental fueron utilizados un total de 20 indicadores. Todos ellos, poseen una carga superior a 0,7, que es el umbral mínimo. Por lo tanto, estos constructos de primer orden están bien medidos por los itms considerados.

Los ítems considerados en el constructo desempeño empresarial también poseen una carga superior a 0,7. Por tanto, está bien medido. 
Tabla 2. Fiabilidad individual de ítems

\begin{tabular}{|lc|}
\hline Constructo/ ítem/ Carga $(\lambda)$ & \\
\hline INNOVACIÓN (constructo reflectivo de primer orden) & 0,717 \\
\hline INN1 & 0,882 \\
INN2 & 0,831 \\
\hline INN3 & \\
\hline PROACTIVIDAD (constructo reflectivo de primer orden) & 0,819 \\
\hline PROACT1 & 0,780 \\
PROACT2 & 0,779 \\
\hline PROACT3 & \\
\hline ASUNCIÓN DE RIESGOS (constructo reflectivo de primer orden) & 0,789 \\
\hline ASUNC RIES1 & 0,858 \\
ASUNC RIES2 & 0,848 \\
\hline ASUNC RIES3 & \\
\hline DESEMPEÑO EMPRESARIAL (constructo reflectivo de primer orden) & 0,801 \\
\hline DESEMP1 & 0,812 \\
DESEMP2 & 0,828 \\
DESEMP3 & 0,816 \\
\hline DESEMP4 & \\
\hline
\end{tabular}

\section{Fiabilidad de los constructos}

Con esta medida tratamos de evaluar la consistencia interna de los indicadores que componen cada constructo. Esto es, si las variables observables están midiendo rigurosamente la variable latente que representan. Para llevar a cabo esta evaluación, podemos utilizar dos criterios: el alpha de cronbach y la fiabilidad compuesta $\left(\rho_{c}\right)^{2}$. La fiabilidad compuesta puede ser un criterio más completo, pues parte de las cargas factoriales reales de los ítems que han sido utilizados en el modelo causal son iguales a la unidad (Fornell y Larcker, 1981; Barclay et al., 1995). Como criterio se acepta como umbral mínimo el valor de 0,7 (Fornell y Larcker, 1981).

2.- La fiabilidad compuesta $(p c)$ se calcula con la siguiente fórmula: $\left(\mathrm{p}_{\mathrm{c}}\right)=\frac{\left(\sum \lambda_{i}\right)^{2}}{\left(\sum \lambda_{i}\right)^{2}+\sum_{i} \operatorname{var}\left(\varepsilon_{i}\right)}$
Fuente: Werts et al. (1974) 


\section{Tabla 3. Alpha de Cronbach y fiabilidad compuesta $\left(\rho_{c}\right)$ de los constructos}

\begin{tabular}{|l|c|c|c|c|}
\hline & Innovación & Proatividad & $\begin{array}{c}\text { Asunción } \\
\text { de Riesgos }\end{array}$ & $\begin{array}{c}\text { Desempeño } \\
\text { Empresarial }\end{array}$ \\
\hline ALPHA DE CRONBACH & 0,748 & 0,706 & 0,777 & 0,833 \\
FIABILIDAD COMPUESTA $\left(\rho_{\mathrm{C}}\right)$ & 0,853 & 0,835 & 0,871 & 0,887 \\
\hline & Dimensión & Dimensión & Dimensión & \\
& Económica & Social & Medioambiental & \\
\hline ALPHA DE CRONBACH & 0,870 & 0,721 & 0,763 & \\
FIABILIDAD COMPUESTA $\left(\rho_{\mathrm{c}}\right)$ & 0,911 & 0,853 & 0,896 & \\
\hline
\end{tabular}

Como podemos comprobar, los valores del alpha de cronbach y la fiabilidad compuesta $\left(\rho_{c}\right)$ (tabla 3) son superiores a 0,7 lo que nos permite comprobar la consistencia interna de todos los indicadores. Estas dos medidas nos permiten afirmar que se está midiendo las variables manifiestas de la misma variable latente de manera rigurosa.

\section{Validez convergente}

Con esta prueba, se trata de medir si un conjunto de indicadores miden un constructo determinado o están midiendo otro concepto distinto. Para analizar este tipo de validez se utiliza la varianza extraída media (AVE3). El criterio de aceptación consiste en que la (AVE) de un constructo debe ser mayor de 0,5 (Fornell y Larcker, 1981). Cuando esto ocurre, el constructo comparte más de la mitad de su varianza con sus indicadores, siendo el resto de la varianza debida al error de la medida. La validez convergente sólo puede ser aplicada a los constructos con indicadores reflectivos, como es nuestro caso. Como podemos observar, todos los AVE de la tabla 4 tienen valores superiores a 0,5. Esto significa que los ítems seleccionados representan de manera adecuada al constructo subyacente (Henseler et al., 2009).

\section{Tabla 4. AVE de los constructos}

\begin{tabular}{|c|c|c|c|c|}
\hline & Innovación & Proatividad & $\begin{array}{c}\text { Asunción } \\
\text { de Riesgos }\end{array}$ & $\begin{array}{c}\text { Desempeño } \\
\text { Empresarial }\end{array}$ \\
\hline AVE & 0,660 & 0,629 & 0,692 & 0,662 \\
\hline & $\begin{array}{c}\text { Dimensión } \\
\text { Económica }\end{array}$ & $\begin{array}{c}\text { Dimensión } \\
\text { Social }\end{array}$ & $\begin{array}{c}\text { Dimensión } \\
\text { Medioambiental }\end{array}$ & \\
\hline AVE & 0,752 & 0,620 & 0,745 & \\
\hline
\end{tabular}

3.- La varianza extraida media ha sido calculada con la siguiente fórmula: Fuente: Fornell \& Larcker (1981)

$$
A V E=\frac{\sum_{i=1}^{p} \lambda_{i}^{2}}{\sum_{i=1}^{p} \lambda_{i}^{2}+\sum_{i=1}^{p} \theta_{\Delta i}}
$$




\section{$\underline{\text { Validez discriminante }}$}

Esta medida nos indica lo diferentes que son los constructos considerados. Existen diferentes métodos para calcular la validez discriminante, aunque nosotros utilizamos el propuesto por Fornell y Larcker (1981). Un constructo estará dotado de validez discriminante cuando la AVE de dicho constructo es mayor que las correlaciones al cuadrado entre ese constructo y los demás que forman el modelo. En la tabla 5 se ha puesto en negrilla los valores de AVE de cada constructo y podemos observar que son mayores a las correlaciones entre cada constructo y el resto.

\section{Tabla 5. Validez discriminante}

\begin{tabular}{|l|c|c|c|c|c|c|c|}
\hline & Innov & Proact & Asunc & $\begin{array}{c}\text { Dimensión } \\
\text { Económica }\end{array}$ & $\begin{array}{c}\text { Dimensión } \\
\text { Social }\end{array}$ & $\begin{array}{c}\text { Dimensión } \\
\text { Medioambiental }\end{array}$ & $\begin{array}{c}\text { Desempeño } \\
\text { Empresarial }\end{array}$ \\
\hline Innov & $\mathbf{0 , 6 6 0}$ & & & & & & \\
Proact & 0,563 & $\mathbf{0 , 6 2 9}$ & & & & & \\
Asunc & 0,603 & 0,490 & $\mathbf{0 , 6 9 2}$ & & & & \\
Dimensión Económica & 0,465 & 0,384 & 0,412 & $\mathbf{0 , 7 5 2}$ & & & \\
Dimensión Social & 0,432 & 0,302 & 0,372 & 0,463 & $\mathbf{0 , 6 2 0}$ & & \\
Dimensión Medioambiental & 0,273 & 0,432 & 0,466 & 0,589 & 0,484 & $\mathbf{0 , 7 4 5}$ & \\
Desempeño Empresarial & 0,521 & 0,511 & 0,525 & 0,543 & 0,465 & 0,497 & $\mathbf{0 , 6 6 2}$ \\
\hline
\end{tabular}

\section{Análisis y evaluación del modelo de medida de los constructos de segundo orden}

En el modelo, hemos considerado dos variables de segundo orden: la orientación emprendedora y la responsabilidad social empresarial. En ambos casos, hemos seguido el método en dos pasos a través de los latent variable scores (Wright et al., 2012). El constructo orientación emprendedora es un constructo formativo y el constructo responsabilidad social empresarial es reflectivo.

Para la validación del constructo orientación emprendedora se tuvo en cuenta las recomendaciones de Diamantopoulos et al. (2008). En este caso, es necesario e imprescindible que los indicadores de cada constructo no presenten problemas de colinealidad (Diamantopoulos y Wilkholver, 2001). Pueden existir problemas de colinalidad si el FIV (factor de inflación de la varianza) es mayor que 5 (Kleinbaum et al., 1988). En nuestro caso, no hay problemas de colinealidad. 


\section{Tabla 6. Estadísticos de colinealidad}

\begin{tabular}{|l|lcc|}
\hline Constructo & Factor & Pesos $(\lambda)$ & FIV \\
\hline ORIENTACIÓN & INNOVACIÓN & 0,362 & 1,381 \\
EMPRENDEDORA & PROACTIVIDAD & 0,464 & 1,664 \\
& ASUNCIÓN DE RIESGOS & 0,449 & 1,282 \\
\hline
\end{tabular}

También debemos realizar un análisis la correlación canónica, que proporciona información sobre la importancia relativa de cada indicador en la formación del constructo en cuestión. Este análisis lo realizamos a partir de los pesos de cada indicador (Chin, 1998). Vemos que todos son positivos y nos indican que la proactividad es la dimensión que más contribuye a formar la orientación emprendedora.

La RSE es constructo de segundo orden reflectivo, por lo que debemos comprobar la fiabilidad individual de las dimensiones consideradas (dimensión económica, dimensión social y dimensión medioambiental). En este caso, todas las cargas, están por encima de 0,5 (Barclay et al., 1995; Chin, 1998).

\section{Tabla 7. Fiabilidad individual de dimensiones de RSE}

\begin{tabular}{|lc|}
\hline Dimensión & Carga $(\lambda)$ \\
\hline Dimensión Social & 0,763 \\
Dimensión Medioambiental & 0,796 \\
Dimensión Económica & 0,876 \\
\hline
\end{tabular}

La fiabilidad del constructo RSE viene dada por el alhpa de Cronbach y fiabilidad compuesta( $\mathrm{c}$ ), que logran valores de 0,870 y 0,911 respectivamente, superando los límites marcados por Fornell y Larcker (1981). La validez convergente del RSE también se da, ya que la AVE es de 0,721, por encima del límite propuesto por Fornell y Larcker (1981).

\section{Evaluación del modelo estructural}

Una vez demostradas la fiabilidad y la validez convergente y discriminante del modelo de medida, se procedió a testar las relaciones entre las distintas variables, comenzando por obtener, mediante el método bootstrap (5000 submuestras), los distintos parámetros estadísticos. El soporte de las hipótesis planteadas se realizó a partir del signo, el valor y la significatividad de los t-valores en cada uno de los coeficientes path (ß). 


\section{Tabla 8. Parámetros asociados al contraste de hipótesis}

\begin{tabular}{|l|ccc|}
\hline Hipótesis & (B) & t-valor & Confirmación \\
\hline $\begin{array}{l}\mathrm{H}_{1}: \text { La orientación emprendedora afecta positivamente al } \\
\text { desempeño empresarial de las cooperativas agroalimentarias. }\end{array}$ & 0,496 & $6,923^{\star \star \star}$ & $\mathrm{Si}$ \\
\hline $\begin{array}{l}\mathrm{H}_{2}: \text { La orientación emprendedora influye positivamente en la RSE } \\
\text { de las cooperativas agroalimentarias. }\end{array}$ & 0,648 & $8,397^{\star \star *}$ & $\mathrm{Si}$ \\
\hline $\begin{array}{l}\mathrm{H}_{3}: \text { La adopción de medidas de RSE en todas sus dimensiones } \\
\text { tiene una influencia positiva en el desempeño empresarial de las } \\
\text { cooperativas agroalimentarias. }\end{array}$ & 0,791 & $9,247^{\star \star *}$ & $\mathrm{Si}$ \\
\hline${ }^{* \star} \mathrm{p}<0.001$ & & & \\
\hline
\end{tabular}

Como podemos comprobar, las tres hipótesis quedan confirmadas (los coeficientes path son positivos, mayores que 0,2 y los valores de la t indican un alto nivel de significación.

Si consideramos la relación directa de la orientación emprendedora en las cooperativas agroalimentarias es capaz de explicar el $24,6 \%$ de la varianza del desempeño empresarial $\left(R^{2}=0,246\right)$.

\section{Figura 2. Modelo de influencia directa de la orientación emprendedora en el desempeño empresarial de las cooperativas agroalimentarias}

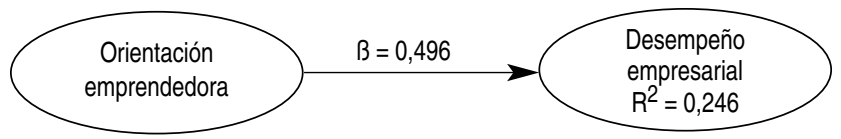

En este trabajo también tratamos de analizar el papel mediador de la RSE en la relación entre la orientación emprendedora y el desempeño empresarial.

\section{Figura 3. Modelo de efecto mediador de la RSE en la relación entre la orientación emprendedora en el desempeño empresarial de las cooperativas agroalimentarias}

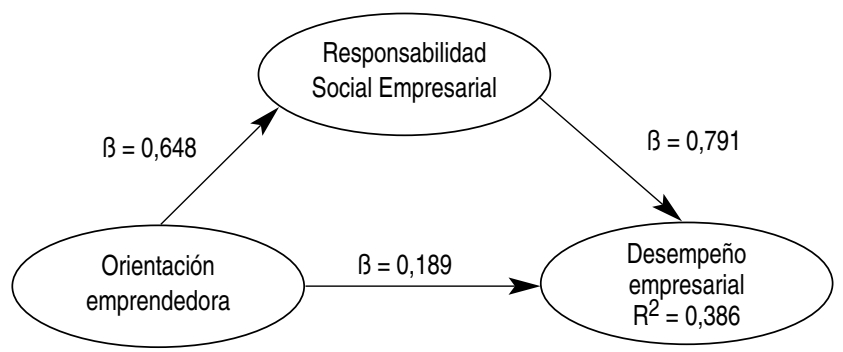




\section{EL EFECTO MEDIADOR DE LA RSE EN LA RELACIÓN DE LA ORIENTACIÓN EMPRENDEDORA Y EL DESEMPEÑO DE LAS COOPERATIVAS AGROALIMENTARIAS}

El segundo objetivo de este trabajo es analizar el efecto de la RSE como variable mediadora en la relación entre la orientación emprendedora y el desempeño empresarial de las cooperativas agroalimentarias. En la figura 3 podemos observar que el efecto indirecto es positivo y significativo (entre orientación emprendedora y RSE $\mathrm{H}_{2}$ : $\beta=0,648 ; p<0,001$; y entre RSE y desempeño empresarial $\mathrm{H}_{3}$ : $\beta=0,791 ; p<0,001$ ). En dicha figura también observamos que el efecto directo (entre orientación emprendedora y desempeño empresarial $\mathrm{H}_{1}$ : 0,189) es suprimido (Hair et al., 2014 lo denomina como efecto supresor, que puede analizarse a partir de la varianza explicada (VAF): si ésta es mayor que 1(en nuestro modelo la VAF obtiene un valor de 1,23), el efecto directo es eliminado. En el modelo que aparece en la figura 3, la relación entre orientación emprendedora y desempeño empresarial, aunque positiva, está por debajo de 0,2 y no es significativa. En definitiva, según Barón y Kenny (1986) se produce mediación de la RSE en la relación entre la orientación emprendedora y el desempeño empresarial de las cooperativas agroalimentarias. Llegados a este punto, el siguiente paso será calificar la mediación. Según los datos que se desprenden de la figura 3, la mediación es total: Además, la evaluación del modelo estructural presenta un buen $\mathrm{GoF}(0,62)$, una consistencia moderada $\left(\mathrm{R}^{2}=\right.$ $0,386)$ y una relevancia predictiva alta $\left(Q^{2} R S E=0,29 ; Q^{2}\right.$ Desempeño empresarial: 0,37$)$.

\section{5.- Conclusiones}

La orientación emprendedora y la responsabilidad social empresarial siguen suscitando el interés académico y empresarial. Este interés también se observa en las cooperativas agroalimentarias.

Desde el punto de vista metodológico, combinación de tres vías para la obtención de datos permite obtener rigurosidad y robustez a la investigación realizada: cuestionario para obtener información sobre orientación emprendedora; memorias de sostenibilidad para los datos de RSE y la base de datos $\mathrm{SABI}$ para los datos económicos objetivos.

Del análisis de los datos, se desprende que el desempeño de las cooperativas agroalimentarias depende de la orientación emprendedora que éstas posean. Hasta tal punto es importante, que la orientación emprendedora es capaz de explicar el $24,6 \%$ de la varianza del desempeño empresarial de las cooperativas agroalimentarias. De las tres dimensiones consideradas como integrantes de la orientación emprendedora, la proactividad es la más relevante: así se desprende del análisis de los efectos indirectos a través del programa SmartPLS 3.2.1.

Analizando el efecto medidor de la RSE en la relación entre la orientación emprendedora y el desempeño, comprobamos que dicho efecto es total: por lo tanto anula el efecto directo y es capaz de 
mejorar la explicación del desempeño de las cooperativas agroalimentarias: con este efecto mediador es posible explicar el 38,6\% de la varianza. La anterior conclusión la obtenemos a través del diseño de una modelo estructural adecuado $(\mathrm{GoF}=0,62)$, que además posee una relevancia predictiva tanto de la RSE como del desempeño empresarial.

Como conclusión final, podemos afirmar que el modelo presenta una consistencia moderada, que viene dada por el $R^{2}=0,386$.

Como futuras líneas de investigación, se podría efectuar un análisis cualitativo de los datos a través del método fuzzy. También sería muy interesante comparar lo que pasa con dicho efecto mediador con otro tipo de cooperativas e incluso con empresas de mercado. Finalmente, dada la globalización de la economía, se podrían desarrollar investigaciones que comparasen este efecto mediador de la responsabilidad social de la empresa en diferentes países con elementos culturales, sociales e institucionales diferenciados.

\section{6.- Bibliografía}

ALLOUCHE, J. \& LAROCHE, P. (2005): "A Meta-Analytical Investigation of the Relationship between Corporate Social and Financial Performance", Revue de Gestion des Ressources Humaines, 57, 18-41.

ALOULOU, W. \& FAYOLLE, A. (2005): "A conceptual approach of entrepreneurial orientation within small business context", Journal of Enterprising Culture, 13 (01), 21-45.

ÁLVAREZ, S. \& BUSENITZ, L. (2001): "The entrepreneurship of resource-based theory", Journal of Management, 27, 755- 775.

ARCAS, N. \& BRIONES, A.J. (2009): "Responsabilidad Social Empresarial de las Organizaciones de la Economía Social. Valoración de la misma en las empresas de la Región de Murcia", CIRIECEspaña, Revista de Economía Pública, Social y Cooperativa, 65, 143-161.

ARCHEL, P., HUSILLOS, J., LARRINAGA, C. \& SPENCE, C. (2009): "Social disclosure, legitimacy theory and the role of the state", Accounting, auditing \& accountability journal, 22 (8), 1284-1307.

ARMSTRONG, J.S. \& OVERTON, T.S. (1977): "Estimating nonresponse bias in mail surveys", Journal of marketing research, 396-402.

BANSAL, P. (2005): "Evolving Sustainably: A Longitudinal Study of corporate Sustainable Development", Strategic Management Journal, 26, 197-218. 


\section{EL EFECTO MEDIADOR DE LA RSE EN LA RELACIÓN DE LA ORIENTACIÓN EMPRENDEDORA Y EL DESEMPEÑO DE LAS COOPERATIVAS AGROALIMENTARIAS}

BARCLAY, D., HIGGINS, C. \&THOMPSON, R. (1995): "The Partial Least Squares (PLS) approach to causal modeling, personal computer adoption and use as an illustration", Technology Studies, special issue on Research Methodology, 2, 285-309.

BARNETT, M. \& SALOMON, R.M. (2012): "Does it Pay to be Really Good? Addressing the Shape of the Relationship between Social and Financial Performance", Strategic Management Journal, 33 (11), 1304-1320.

BARNETT, M.L. \& SALOMON R.M. (2006): "Beyond Dichotomy: The Curvilinear Relationship Between Social Responsibility and Financial Performance", Strategic Management Journal, 27 (11), 1101.

BARROSO, C., CARRIÓN, G.C. \& ROLDÁN, J.L. (2010): "Applying maximum likelihood and PLS on different sample sizes: studies on SERVQUAL model and employee behavior model". In: Handbook of partial least squares, Springer Berlin Heidelberg, 427-447.

BASSO, O., FAYOLLE, A. \& BOUCHARD, V. (2009): "Entrepreneurial orientation: the making of a concept", The International Journal of Entrepreneurship and Innovation, 10(4), 313-321.

BOSMA, N.S. \& LEVIE, J. (2010): Global Entrepreneurship Monitor 2009 Executive Report.

CARMINES, E.G. \& ZELLER, R.A. (1979): Realiability and Validity Assessment, Sage University Paper Series on Quantitative apverly Hills.

CARRASCO, I. (2007): "Corporate social responsibility, values, and cooperation", International Advances in Economic Research, 13 (4), 454-460.

CARROLL, A.B. (1999): "Corporate social responsibility: evolution of a definitional construct", Business \& Society, 38 (3), 268-295.

CARROLL, A.B. \& SHABANA, K.M. (2010): "The business case for corporate social responsibility: a review of concepts, research and practice", International Journal of Management Reviews, 12 (1), 85-105.

CHANDRA, Y., STYLES, C. \& WILKINSON, I. (2009): "The recognition of first time international entrepreneurial opportunities: Evidence from firms in knowledge-based industries", International Marketing Review, 26(1), 30-61.

CHAVAN, M. (2005): "An appraisal of environment management systems: A competitive advantage for small businesses", Management of Environmental Quality, 16 (5), 444-463.

CHIN, W.W. (1998): "The Partial Least Squares Approach to Structural Equation Modeling". In: G.A. Marcoulides (Ed), Modern Methods for Business Research, Mahwah NJ: Lawrence Erlbaum Associates, Publisher, 295-336.

CHIRICO, F., SIRMON, D.G., SCIASCIA, S. \& MAZZOLA, P. (2011): "Resource orchestration in family firms: Investigating how entrepreneurial orientation, generational involvement, and participative strategy affect performance", Strategic Entrepreneurship Journal, 5(4), 307-326. 
CHOW, W.S. \& CHEN, Y. (2012): "Corporate Sustainable Development: Testing a New Scale Based on the Mainland Chinese Context", Journal of Business Ethics, 105, 519-533.

CONCA, F.J., LLOPIS, J. \& TARI, J.J. (2004): "Development of a measure to assess quality management in certified firms", European journal of operational research, 156 (3), 683-697.

COVIN, J.G. \& LUMPKIN, G.T. (2011): "Entrepreneurial orientation theory and research: Reflections on a needed construct", Entrepreneurship Theory and Practice, 35 (5), 855-872.

COVIN, J.G. \& MILLER, D. (2014): "International entrepreneurial orientation: Conceptual considerations, research themes, measurement issues, and future research directions", Entrepreneurship Theory and Practice, 38 (1), 11-44.

COVIN, J.G. \& SLEVIN, D.P. (1989): "Strategic management of small firms in hostile and benign environments", Strategic management journal, 10 (1), 75-87.

COVIN, J.G. \& SLEVIN, D.P. (1991): "A conceptual model of entrepreneurship as firm behavior", Entrepreneurship: Critical perspectives on business and management, 3.

DAHLSRUD, A. (2008): "How corporate social responsibility is defined: an analysis of 37 definitions", Corporate social responsibility and environmental management, 15 (1), 1.

DE CASTRO SANZ, M. (2005): "La Responsabilidad Social de las Empresas, o un nuevo concepto de empresa", CIRIEC-España, Revista de Economía Pública, Social y Cooperativa, 53, 29-51.

DE LA CUESTA GONZÁLEZ, M. (2004): "El porqué de la responsabilidad social corporativa", Boletín económico de ICE, Información Comercial Española, 2813, 45-58.

DE LA CUESTA GONZÁLEZ, M., VALOR MATÍNEZ, C. \& KREISLER MERINO, I. (2003): "Promoción institucional de la Responsabilidad Social Corporativa: iniciativas internacionales y nacionales", Boletín ICE Económico: Información Comercial Española, 2779, 9-20.

DESS, G.G. \& LUMPKIN, G.T. (2005): "The role of entrepreneurial orientation in stimulating effective corporate entrepreneurship", The Academy of Management Executive, 19 (1), 147-156.

DIAMANTOPOULOS, A., RIEFLER, P. \& ROTH, K.P. (2008): "Advancing formative measurement models", Journal of Business Research, 61 (12), 1203-1218.

DIAMANTOPOULOS, D. \& WINKLHOFER, H. (2001): "Index Construction with Formative Indicators: An Alternative to Scale Development", Journal of Marketing Research, 38 (2), 269-277.

DIMITRATOS, P., LIOUKAS, S. \& CARTER, S. (2004): "The relationship between entrepreneurship and international performance: the importance of domestic environment", International Business Review, 13 (1), 19-41.

ELKINGTON, J. (2004): "Enter the Triple Bottom Line". In: A. Henriques \& J. Richardson (Eds.), The Triple Bottom Line, does it all add up?: assessing the sustainability of business and CSR, London, UK: Earthscan, 1-16. 


\section{EL EFECTO MEDIADOR DE LA RSE EN LA RELACIÓN DE LA ORIENTACIÓN EMPRENDEDORA Y EL DESEMPEÑO DE LAS COOPERATIVAS AGROALIMENTARIAS}

EPSTEIN, M.J. BUHOVAC, A.R. \& YUTHAS, K. (2012): Managing social, environmental and financial performance simultaneously, Long Range Planning.

ETCHEBARNE, M.S., GELDRES, V.V. \& GARCÍA, R. (2010): "The impact of entrepreneurial orientation on firms export performance", ESIC Market Economic and Business Journal, 137, 165-191.

FORNELL, C. \& LARCKER, D.F. (1981): "Evaluating Structural Equation Models with Unobservable Variables and Measurement Error", Journal of marketing research, 18, 39-50

GALAN, J.I. \& SANCHEZ-BUENO, M.J. (2009): "The Continuing Validity of the Strategic-Structure Nexus; New Findings, 1993-2003", Strategic Management Journal, 30 (11), 1234-1243.

GALLARDO-VÁZQUEZ, D., SÁNCHEZ-HERNÁNDEZ, M.I. \& CORCHUELO-MARTÍNEZ-AZÚA, M.B. (2013): "Validación de un instrumento de medida para la relación entre la orientación a la Responsabilidad Social Corporativa y otras variables estratégicas de la empresa", Revista de Contabilidad, 16(1), 11-23.

GARRIGA, E. \& MELÉ, D. (2004): "Corporate Social Responsibility Theories: Mapping the Territory", Journal of Business Ethics, 53, 51-71.

GARRIGA, E. \& MELÉ, D. (2004): Ética empresarial.

GEFEN, D., STRAUB, D.W. \& RIGDON, E.E. (2011): "An update and extension to SEM guidelines for admnistrative and social science research", Management Information Systems Quarterly, 35 (2), iii-xiv.

GÓMEZ, F. (2008): Responsabilidad social corporativa y performance financiera: Treinta y cinco años de investigación empírica en busca de un consenso", Principios: Estudios de Economía Política, 11, 5-22.

GRIFFIN, J.J. \& MAHON, J.F. (1997): "The Corporate Social Performance and Corporate Financial Performance Debate. Twenty-five Years of Incomparable Research. Business and Society", 36 (1), 5-31.

HAIR, J.F., HULT, G.T.M., RINGLE, C.M. \& SARSTEDT, M. (2014): A Primer on Partial Least Squares Structural Equation Modeling (PLSSEM), Thousand Oaks, CA: Sage.

HENSELER, J. \& SARSTEDT, M. (2013): "Goodness-of-fit indices for partial least squares path modeling", Computational Statistics, 28 (2), 565-580.

HENSELER, J., RINGLE, C.M. \& SINKOVICS, R.R. (2009): "The Use of Partial Least quares Path Modeling in International Marketing", Advances in International Marketing, 20, 277-320.

HERNÁNDEZ-PERLINES, F. (2014): "Orientación emprendedora de las cooperativas agroalimentarias con actividad exportadora", CIRIEC-España, Revista de Economía Pública, Social y Cooperativa, 80, 29-56. 
HERNÁNDEZ-PERLINES, F. \& SÁNCHEZ-INFANTE HERNÁNDEZ, J.P. (2015): "La influencia de la responsabilidad social empresarial (RSE) en los resultados económicos (RE) de las micro, pequeñas y medianas empresas (MIPYMES)". En: XXIII Congreso EBEN España, Universidad Pablo de Olavide de Sevilla.

HUBER, G.P. \& POWER, D.J. (1985): "Research notes and communications retrospective reports of strategic-level managers: Guidelines for increasing their accuracy", Strategic Management Journal (pre-1986), 6 (2), 171.

HULT, G.T.M., SNOW, C.C. \& KANDEMIR, D. (2003): "The role of entrepreneurship in building cultural competitiveness in different organizational types", Journal of management, 29 (3), 401-426.

HUSTED, B.W., \& ALLEN, D.B. (2000): "Is it ethical to use ethics as strategy?". In: Business Challenging Business Ethics: New Instruments for Coping with Diversity in International Business Springer Netherlands, 21-31.

IRELAND, R.D., HITT, M.A. \& SIRMON, D.G. (2003): "A model of strategic entrepreneurship: The construct and its dimensions", Journal of management, 29 (6), 963-989.

KLEINBAUM, D.G. KUPPER, L.L., MULLER, K.E. \& NIZAM, A. (1988): "One-way analysis of variance", In: Applied regression analysis and other multivariable methods, PWS-Kent Publishing Co Boston, 341-386.

KNIGHT, G.A. (1997): "Cross-cultural reliability and validity of a scale to measure firm entrepreneurial orientation", Journal of business venturing, 12 (3), 213-225.

KNIGHT, G.A. \& CAVUSGIL, S.T. (2005): "A taxonomy of born-global firms", MIR: Management International Review, 15-35.

KRAUS, S., RIGTERING, J.C., HUGHES, M. \& HOSMAN, V. (2012): "Entrepreneurial orientation and the business performance of SMEs: a quantitative study from the Netherlands", Review of Managerial Science, 6 (2), 161-182.

KROPP, F., LINDSAY, N.J. \& SHOHAM, A. (2008): "Entrepreneurial orientation and international entrepreneurial business venture startup", International Journal of Entrepreneurial Behavior \& Research, 14 (2), 102-117.

KUIVALAINEN, O., PUUMALAINEN, K., SINTONEN, S. \& KYLÄHEIKO, K. (2010): "Organisational capabilities and internationalisation of the small and medium-sized information and communications technology firms", Journal of International Entrepreneurship, 8 (2), 135-155.

KURATKO, D.F. \& AUDRETSCH, D.B. (2009): "Strategic entrepreneurship: exploring different perspectives of an emerging concept", Entrepreneurship Theory and Practice, 33 (1), 1-17.

LARRÁN, M., HERRERA, J. \& LECHUGA, M.P. (2011): "El estado del arte en la investigación sobre Responsabilidad Social y PYMES". En: XVI congreso AECA Nuevos Modelos Económicos: Empresa, Mercados y Culturas, Granada, España, 21-23 Septiembre. 


\section{EL EFECTO MEDIADOR DE LA RSE EN LA RELACIÓN DE LA ORIENTACIÓN EMPRENDEDORA Y EL DESEMPEÑO DE LAS COOPERATIVAS AGROALIMENTARIAS}

LUMPKIN, G.T. \& DESS, G.G. (1996): "Clarifying the entrepreneurial orientation construct and linking it to performance", Academy of management Review, 21(1), 135-172.

LYON, D.W., LUMPKIN, G.T. \& DESS, G.G. (2000): "Enhancing entrepreneurial orientation research: Operationalizing and measuring a key strategic decision making process", Journal of management, 26 (5), 1055-1085.

MARGOLIS, J.D., ELFENBEIN, H.A. \& WALSH, J.P. (2007): "Does it pay to be good? A meta-analysis and redirection of research on the relationship between corporate social and financial performance", Academy of Management Meetings, Philadelphia, United States, August.

MARÍN, L. \& RUBIO, A. (2008): "¿Moda o factor competitivo? Un estudio empírico de responsabilidad social corporativa en Pyme", Información Comercial Española, 842, 177-193.

MAROM, I.Y. (2006): "Toward a unified theory of the CSP-CFP link", Journal of Business Ethics, 67 (2), 191-200.

MARTíNEZ-CAMPILLO, A., CABEZA-GARCÍA, L. \& MARBELLA-SÁNCHEZ, F. (2013): "Responsabilidad social corporativa y resultado financiero: evidencia sobre la doble dirección de la causalidad en el sector de las Cajas de Ahorros", Cuadernos de Economía y Dirección de la Empresa, 16 (1), 54-68.

McWILLIAMS, A. \& SIEGEL, D. (2001): "Corporate Social Responsibility: A Theory of the Firm Perspective", The Academy of Management Review, 26 (1), 117-127.

MILLER, D. (1983): "The correlates of entrepreneurship in three types of firms", Management science, 29 (7), 770-791.

MILLER, D. (2011): "Miller (1983) revisited: A reflection on EO research and some suggestions for the future", Entrepreneurship Theory and Practice, 35 (5), 873-894.

MILLER, D. \& FRIESEN, P.H. (1983): "Strategy making and environment: the third link", Strategic management journal, 4 (3), 221-235.

MIRAS, M.D.M., CARRASCO GALLEGO, A. \& ESCOBAR, B. (2014): "Responsabilidad Social Corporativa y rendimiento financiero: un meta-análisis", Revista Española de Financiación y Contabilidad, 43 (2), 193-215.

MISHRA, S. \& SUAR, D. (2010): "Does corporate social responsibility influence firm performance of Indian companies?", Journal of Business Ethics, 95 (4), 571-601.

MOORE, G. (2001): "Corporate Social and Financial Performance: An Investigation in the U.K.Supermarket Industry", Journal of Business Ethics, 34, 299-315.

MORENO, A.M. \& CASILLAS, J.C. (2008): "Entrepreneurial orientation and growth of SMEs: A causal model", Entrepreneurship Theory and Practice, 32 (3), 507-528. 
MORRIS, M.H., KURATKO, D.F., SCHINDEHUTTE, M. \& SPIVACK, A.J. (2012): "Framing the entrepreneurial experience", Entrepreneurship Theory and Practice, 36 (1), 11-40.

MOZAS, A. \& PUENTES, R. (2010): "La responsabilidad social corporativa y su paralelismo con las sociedades cooperativas", REVESCO, Revista de Estudios Cooperativos, 103, 75-100.

NALDI, L., NORDQVIST, M., SJÖBERG, K. \& WIKLUND, J. (2007): "Entrepreneurial orientation, risk taking, and performance in family firms", Family business review, 20 (1), 33-47.

NIETO ANTOLÍN, M. \& FERNÁNDEZ GAGO, R. (2004): "Responsabilidad social corporativa: la última innovación en management", Universia Business Review, 1, 28-39.

ORLITZKY, M., SCHMIDT, F.L. \& RYNES, S.L. (2003): "Corporate social and financial performance: A meta-analysis", Organization studies, 24 (3), 403-441.

PÉREZ-SÁNCHEZ, D., BARTON, J.R. \& BOWER, D. (2003): "Implementing environmental management in SMEs", Corporate Social Responsibility \& Environmental Management, 10 (2), 67-77.

PHILLIPS, R., FREEMAN, R.E. \& WICKS, A.C. (2003): "What stakeholder theory is not", Business Ethics Quarterly, 13 (04), 479-502.

PORTER, M.E. \& KRAMER, M.R. (2006): "Estrategia y sociedad", Harvard Business Review, 84 (12), 42-56.

PUENTES POYATOS, R., VELASCO GÁMEZ, M.D. \& VILAR HERNÁNDEZ, J. (2009): "El buen gobierno corporativo en las sociedades cooperativas", REVESCO, Revista de Estudios Cooperativos, 98, 118-140.

RAUCH, A., WIKLUND, J., LUMPKIN, G.T. \& FRESE, M. (2009): "Entrepreneurial orientation and business performance: An assessment of past research and suggestions for the future", Entrepreneurship Theory and Practice, 33 (3), 761-787.

REINARTZ, W., HAENLEIN, M. \& HENSELER, J. (2009): "An empirical comparison of the efficacy of covariance-based and variance-based SEM", International Journal of research in Marketing, 26 (4), 332-344.

REVELL, A., STOKES, D. \& CHEN, H. (2010): "Small Businesses and the Environment: Turning over a new leaf?", Business Strategy and the Environment, 19 (5), 273-288.

RINGLE, C.M., WENDE, S. \& BECKER, J.M. (2015): Smart PLS 3. Boenningstedt: SmartPLS GmbH.

RINGLE, C.M., WENDE, S. \& WILL, A. (2010): "Finite mixture partial least squares analysis: Methodology and numerical examples". In: Handbook of partial least squares, Springer Berlin Heidelberg, 195-218.

RUIZ-ORTEGA, M.J., PARRA-REQUENA, G., RODRIGO-ALARCÓN, J. \& GARCÍA-VILLAVERDE, P.M. (2013): "Environmental dynamism and entrepreneurial orientation: The moderating role of firm's capabilities", Journal of Organizational Change Management, 26 (3), 475-493. 


\section{EL EFECTO MEDIADOR DE LA RSE EN LA RELACIÓN DE LA ORIENTACIÓN EMPRENDEDORA Y EL DESEMPEÑO DE LAS COOPERATIVAS AGROALIMENTARIAS}

RUNYAN, R., DROGE, C. \& SWINNEY, J. (2008): "Entrepreneurial orientation versus small business orientation: what are their relationships to firm performance?", Journal of Small Business Management, 46 (4), 567-588.

SCHADEWITH, H. \& NISKALA, M. (2010): "Communication via Responsibility Reporting and its Effect on Firm Value in Finland", Corporate Social Responsibility and Environmental Management, 17, 96-106.

SCHAPER, M.T. \& SAVERY, L.K. (2004): "Entrepreneurship and philanthropy: the case of small Australian firms", Journal of Developmental Entrepreneurship, 9(3), 239.

SCHUMPETER, J.A. (1934): The theory of economic development: An inquiry into profits, capital, credit, interest, and the business cycle, Vol. 55, Transaction publishers.

SERVER IZQUIERDO, R.J. \& CAPÓ VICEDO, J.C. (2009): "La Responsabilidad Social Empresarial en un contexto de crisis. Repercusión en las Sociedades Cooperativas", CIRIEC-España, Revista de Economía Pública, Social y Cooperativa, 65, 7-31.

STRANDBERG, L. (2010): "La medición y la comunicación de la RSE: indicadores y normas", Cuadernos de la Cátedra "La Caixa" de Responsabilidad Social de la Empresa y Gobierno Corporativo, Business School, Universidad de Navarra, 9.

SUNDQVIST, S., KYLÄHEIKO, K., KUIVALAINEN, O. \& CADOGAN, J.W. (2012): "Kirznerian and Schumpeterian entrepreneurial-oriented behavior in turbulent export markets", International Marketing Review, 29 (2), 203-219.

SURROCA, J., TRIBÓ, J.A. \& WADDOCK, S. (2010): "Corporate responsibility and financial performance: The role of intangible resources", Strategic Management Journal, 31 (5), 463-490.

TOMOMI, T. (2010): "Environmental Management Strategy for Small and Medium Sized Enterprises: Why do SMBs Practice Environmental management?", Asian Business and Management, 9 (2), 265-280.

TORO, D. (2006): El enfoque estratégico de la responsabilidad social corporativa: revisión de la literatura académica", Intangible Capital, 2 (14), 338-358.

VARGAS SÁNCHEZ, A. \& VACA ACOSTA, R.M. (2005): "Responsabilidad Social Corporativa y cooperativismo: Vínculos y potencialidades", CIRIEC-España, Revista de Economía Pública, Social y Cooperativa, 53, 241-260.

VÁZQUEZ-CARRASCO, R. \& LÓPEZ-PÉREZ, M.E. (2012): Small \& medium-sized enterprises and Corporate Social Responsibility: a systematic review of the literature, Quality and Quantity.

WADDOCK, S.A. \& GRAVES, S.B. (1997): "Corporate social Performance-Financial Performance Link", Strategic Management Journal, 18 (4), 303-319.

WANG, T. \& BANSAL, P. (2012): "Social responsibility in new ventures: profiting from a long term orientation", Strategic Management Journal, 33 (10), 1135-1153. 
WIKLUND, J. \& SHEPHERD, D. (2005): "Entrepreneurial orientation and small business performance: a configurational approach", Journal of business venturing, 20 (1), 71-91.

WILLIAMSON, D., LYNCH-WOOD, G. \& RAMSAY, J. (2006): "Drivers of Environmental Behaviour in Manufacturing SMEs and the Implications for CSR", Journal of Business Ethics, 67 (3), 317-330.

WRIGHT, R.T., CAMPBELLI, D.E., THATCHER, J.B. \& ROBERTS, N. (2012): "Operationalizing Multidimensional Constructs in Structural Equation Modeling: Recommendations for IS Research", Communications of the Association for Information Systems, 30 (1), 23.

WU, M.L. (2006): "Corporate Social Performance, Corporate Financial Performance and Firm Size", Journal of American Academy of Business, Cambridge, 8 (1), 163-171.

YLI RENKO, H., AUTIO, E., \& SAPIENZA, H. J. (2001): "Social capital, knowledge acquisition, and knowledge exploitation in young technology based firms", Strategic management journal, 22 (67), 587-613.

ZAHRA, S.A., IRELAND, R.D. \& HITT, M.A. (2000): "International expansion by new venture firms: International diversity, mode of market entry, technological learning, and performance", Academy of Management journal, 43 (5), 925-950. 